\title{
Evaluación de la hidrólisis alcalina-enzimática para la obtención de colágeno hidrolizado a partir de virutas de cuero curtido
}

\author{
Evaluation of alkaline-enzymatic hydrolysis to obtain \\ hydrolyzed collagen from tanned leather shavings
}

\section{Avaliação da hidrólise alcalina-enzimática para obter de colágeno hidrolisado de aparas de couro curtido}

\author{
Juan Sebastián Vallejo-Rodríguez ${ }^{1 *}$; Leidy Yaneth Almonacid-Jiménez \\ Rafael Nikolay Agudelo-Valencia ${ }^{1}$; Javier Adolfo Hernández-Fernández ${ }^{2}$; Óscar Leonardo Ortiz-Medina ${ }^{1}$ \\ ${ }^{1}$ Facultad de Ingeniería, Universidad Libre, Sede Bosque Popular, Carrera 70 n. ${ }^{\circ}$ 53-40, Bogotá D.C, Colombia. \\ ${ }^{2}$ Universidad Jorge Tadeo Lozano, Carrera 4 No. 22-61, Bogotá D. C., Colombia.
}

*juans-valejor@unilibre.edu.co

Fecha recepción: 16 de marzo de 2019 Fecha aceptación: 27 de junio de 2019

\begin{abstract}
Resumen
Las virutas de cuero curtido representan el residuo sólido generado durante la etapa de rebajado de cueros como parte del curtido y teñido de pieles; este residuo representa un riesgo al ambiente al momento de su disposición final, debido al alto contenido de cromo, por lo cual se hace necesario evaluar alternativas para el tratamiento de este. En el proceso experimental se empleó la hidrólisis enzimática de la viruta para obtener colágeno hidrolizado; las variables o factores de proceso empleadas fueron el $\mathrm{pH}(8 \mathrm{y}$ 10 ), tiempos de reacción (11 a 13 horas) y dosis de enzima BLUE G-Dupont internacional $(0,00001 \mathrm{~kg}$, $0,0000125 \mathrm{~kg}$ y $0,000015 \mathrm{~kg}$ ). Los resultados experimentales determinaron que las condiciones óptimas para la obtención de colágeno hidrolizado en su mayor contenido fueron: $\mathrm{pH} 10$, dosis de enzima de $0,000015 \mathrm{~kg}$ y 12,5 horas de tiempo de reacción para obtener una concentración de colágeno hidrolizado de $1,828 \mathrm{~kg} / \mathrm{m}^{3}$ correspondiente a una recuperación del 9,14 \% en masa con respecto a la masa de viruta de cuero curtido utilizada para cada ensayo.
\end{abstract}

Palabras claves: Colágeno; Hidrólisis Enzimática; Virutas Cuero Curtido.

\begin{abstract}
The tanned leather shavings represent the solid waste generated during the leather cutting stage as part of the tanning and dyeing of skins; this waste represents a risk to the environment at the time of its final disposal, because of the high chromium content, situation that makes it necessary to evaluate alternatives for its treatment. In the experimental process, the enzymatic hydrolysis of the chip was used to obtain hydrolyzed collagen; the variables or process factors used were the $\mathrm{pH}$ (8 and 10), reaction times (11 to 13 hours) and dose of BLUE G enzyme-Dupont international $(0.00001 \mathrm{~kg}, 0.0000125 \mathrm{~kg} \mathrm{y}$ $0.000015 \mathrm{~kg}$ ). The experimental results gave rise to determine that the optimal conditions for obtaining hydrolyzed collagen in its highest content were $\mathrm{pH} \mathrm{10,} \mathrm{enzyme} \mathrm{dose} \mathrm{of} 0.000015 \mathrm{~kg}$ and 12.5 hours of reaction time to obtain a hydrolyzed collagen concentration of $1.828 \mathrm{~kg} / \mathrm{m}^{3}$ corresponding to a recovery of $9.14 \%$ by mass with respect to the mass of tanned leather chip used for each test.
\end{abstract}

Keywords: Collagen; Enzymatic Hydrolysis; Leather Shavings.

Cita: Vallejo Rodríguez JS, Almonacid Jiménez LY, Agudelo Valencia RN, Hernández Fernández JA, Ortiz Medina OL. Evaluación de la hidrólisis alcalina-enzimática para la obtención de colágeno hidrolizado a partir virutas de cuero curtido. rev.ion. 2019;32(1):55-62. doi:10.18273/revion.v32n1-2019005 


\section{Resumo}

As aparas de couro curtido representam o resíduo sólido gerado durante o estágio de redução de couro como parte do curtimento e tingimento de peles; este resíduo representa um risco para o meio ambiente no momento de sua disposição final, devido ao alto teor de cromo, situação que torna necessária a avaliação de alternativas para o tratamento desses. No processo experimental, a hidrólise enzimática do chip foi utilizada para obtenção de colágeno hidrolisado, as variáveis ou fatores de processo utilizados foram pH (8 e 10), tempos de reação (11 a 13 horas) e dose da enzima AZUL G -Dupont Internacional $(0,00001 \mathrm{~kg}, 0,0000125 \mathrm{~kg}$ y $0,000015 \mathrm{~kg})$. Os resultados experimentais permitiram determinar que as condições ótimas para obtenção de colágeno hidrolisado em seu maior teor foram pH 10, dose enzimática de $0,000015 \mathrm{~kg}$ e 12,5 horas de tempo de reação para obter uma concentração de colágeno hidrolisado de $1,828 \mathrm{~kg} / \mathrm{m}^{3}$ correspondente a uma recuperação de 9,14 \% em massa em relação à massa de lascas de couro curtido utilizada para cada ensaio.

Palavras-chave: Colagénio; Hidrólise Enzimática; Aparas Couro Curtido.

\section{Introducción}

El sector curtidor tiene como objeto la transformación de piel animal en cuero útil para la elaboración de otros artículos. Este sector actualmente representa uno de los mayores generadores de contaminación dado que la etapa productiva da lugar a múltiples subproductos y residuos, los cuales al ser dispuestos de forma inadecuada perjudican la salud y disminuyen la calidad ambiental [1]. El curtido de pieles bovinas tiene como finalidad lograr que la piel sea un material resistente a la acción del agua, el sol, las bacterias y los esfuerzos mecánicos, con el fin de conformar o confeccionar diferentes accesorios de uso cotidiano. Dentro del proceso de curtido se debe regular el espesor o calibre del cuero de acuerdo con el producto destinado, lo cual da lugar a residuos como viruta de cuero curtido, carnaza y retazos de piel [2], los cuales son empleados comúnmente en diferentes campos de la industria como el calzado, las tejas [3], la cosmética y la farmacéutica; asimismo, estos residuos son utilizados en la elaboración de juguetes y alimentos para mascotas [4], o son desechados de forma convencional e inadecuada. Debido al volumen de virutas de cuero curtido (VCC) generado por el sector y a las características propias que el cuero posee, se hace necesario encontrar alternativas de manejo para la valorización del residuo y la disminución del impacto ambiental generado por la mala disposición del material [5]. Las VCC representan del $20 \%$ al $25 \%$ del total de los residuos generados durante el proceso [6]; la VCC está compuesta por una mezcla de colágeno y cromo entre un $2 \%$ y un 5 $\%$ en masa del material seco [7]. Tanto el colágeno hidrolizado como el cromo pueden ser recuperados mediante la aplicación de alternativas que permitan la extracción o separación de estos para el posterior aprovechamiento en diferentes procesos productivos, lo que permite lograr la valorización económica de los residuos y la mitigación de impactos ambientales. Las virutas de cuero curtido (VCC) representan uno de los residuos que se genera en mayor cantidad dentro del proceso productivo del sector curtidor. Por tal razón, es importante evaluar alternativas para la valorización o aprovechamiento de este residuo y a partir de esto contribuir a la disminución de la contaminación originada por la inadecuada disposición del residuo [8]. Algunos estudios realizados se enfocan en el tratamiento de virutas de cuero curtido (VCC) como alternativa de aprovechamiento y valorización del material mediante la extracción de colágeno hidrolizado a partir del método de hidrólisis alcalinaenzimática [9]-[11], con el propósito de obtener productos para ser empleados en diferentes campos.

El presente estudio evaluó la hidrólisis enzimática [12] como alternativa ambiental para la recuperación de colágeno en su forma hidrolizada a partir de las VCC como opción de tratamiento mediante prácticas ambientales enfocadas en la reducción de la acumulación de residuos, mitigación de la contaminación para la obtención de un producto con mayor valor agregado que a su vez contribuya con la minimización de impactos ambientales negativos hacia la sociedad y el ambiente en busca del desarrollo sostenible del sector curtidor.

\section{Metodología}

\section{Materiales y métodos}

Para el desarrollo del proyecto se empleó un diseño experimental de tipo Box Behnken, en el cual se presentan combinaciones, a partir de los bordes 
experimentales o límites de los factores a evaluar [13]. Como variables o factores de proceso a analizar se evaluó el $\mathrm{pH}$, dosis de enzima BLUE $\mathrm{G}$ y el tiempo de reacción, es decir, 3 factores en 3 niveles de cada variable para un total de 17 experimentos. Los rangos de las variables en estudio empleados para la elaboración de los ensayos fueron $\mathrm{pH}(8$, $9,10)$, dosis de enzima $(0,00001 \mathrm{~kg}, 0.0000125$ $\mathrm{kg}$ y $0,000015 \mathrm{~kg}$ ) y un tiempo de reacción (12, 12,5 y 13 horas), se adicionó $0,001 \mathrm{~kg}$ de VCC en $0,00005 \mathrm{~m}^{3}$ de mezcla acuosa reactiva. La enzima fue suministrada por la empresa MerQuiand, la cual tiene un valor de actividad enzimática de 29.000 GLCU/g (Genencor Leather Carbohydrase Units /grame) [14]. Los ensayos fueron realizados en modo batch y en Beakers de 0,000250 $\mathrm{m}^{3}$. El $\mathrm{pH}$ de las soluciones de reacción o hidrólisis fue determinado con un potenciómetro Metrohm 781 pH/ion meter. La agitación en los ensayos fue realizada en una plancha con agitación magnética RT5 IKA y el pH a lo largo de los ensayos fue ajustado con $\mathrm{NaOH} 1 \mathrm{M}$ marca Merck Millipore.

La cantidad de colágeno hidrolizado obtenido fue la variable de respuesta en los experimentos realizados, para lo cual se ejecutaron los análisis respectivos del método de Bradford y electroforesis en gel de poliacrilamida, con el propósito de determinar en qué muestra o ensayo se encontraría mayor cantidad de colágeno hidrolizado.

Cuantificación de proteína mediante el método de Bradford: De acuerdo con la metodología propuesta para la obtención de colágeno hidrolizado, se procede a implementar el método de Bradford con el fin de cuantificar la proteína presente en las muestras [15]. Se realiza la medición para cada ensayo y por medio de la coloración de la proteína y posterior lectura de absorbancia se determina la concentración mediante la ejecución de la curva patrón con albúmina sérica bovina (BSA), azul de coomassie G-250, reactivo de Bradford y el espectrofotómetro ajustado a $595 \mathrm{~nm}$.

Análisis estadístico de los resultados: En cuanto al análisis estadístico se realizó el análisis de varianza de los resultados y se empleó el método de superficie de respuesta para determinar las mejores condiciones del proceso evaluado. El análisis de varianza (ANOVA) permite evaluar y analizar las diferentes situaciones o alternativas que pueden presentarse en un momento dado [16] al realizar un estudio o comprobar una hipótesis. Por otra parte, el método de superficies de respuesta consiste en el conjunto de técnicas matemáticas y estadísticas que permiten analizar la respuesta de interés mediante la influencia de las variables, con el propósito de llegar a la optimización del funcionamiento de un proceso [17].

Determinación del porcentaje (\%) en masa: Para evidenciar la relación porcentual aproximada de colágeno presente en las VCC, se determinó el valor másico porcentual a partir del colágeno hidrolizado presente en la mezcla de extracción, partiendo de la mayor concentración de colágeno hidrolizado obtenido y de la cantidad de materia prima, es decir, la VCC empleada para la ejecución de cada uno de los ensayos experimentales realizados. Por medio de:

$$
\frac{\text { Concentración de proteína obtenida }\left(\frac{\mathrm{kg}}{\mathrm{m}^{3}}\right) * \text { Volumen de solución empleada }\left(\mathrm{m}^{3}\right)}{\text { Cantidad de VCC empleada }(\mathrm{kg})}
$$

Análisis de electroforesis en gel de SDSpoliacrilamida: Para identificar la concentración presente en el ensayo óptimo, se realizó el análisis de electroforesis en gel de poliacrilamida SDSPAGE [18], en el cual se empleó un gel separador al $12 \%$ y concentrador al $4 \%$ para determinar la pureza del colágeno y su tipo. Para esto se tuvo como referencia tanto el patrón de proteínas OptiProtein XL Marker que abarca concentraciones en un rango de 10-245 kDa [19] como la albúmina sérica bovina BSA de $66 \mathrm{kDa}$ [20].

\section{Resultados y discusión}

La tabla 1 corresponde al análisis de varianza de los resultados obtenidos, se observa que el ensayo para el cual se alcanzó la mayor concentración de proteína fue realizado a $\mathrm{pH}$ de 10 (alcalino), dosis de enzima BLUE G de 0,000015 kg y un tiempo de reacción de 12,5 horas. Bajo estas condiciones, la cantidad de proteína hidrolizada obtenida fue $1,828 \mathrm{~kg} / \mathrm{m}^{3}$. 
Tabla 1. Anova para los ensayos realizados.

\begin{tabular}{cccccc}
\hline \multicolumn{5}{c}{ Análisis de varianza (ANOVA) } \\
\hline $\begin{array}{c}\text { Fuente de } \\
\text { variación }\end{array}$ & $\begin{array}{c}\text { Suma de } \\
\text { cuadrados }\end{array}$ & $\begin{array}{c}\text { Grados de } \\
\text { libertad }\end{array}$ & $\begin{array}{c}\text { Cuadrados } \\
\text { medios }\end{array}$ & $\begin{array}{c}\text { Valor } \\
\mathrm{F}\end{array}$ & Prob > F \\
\hline Modelo & 3,187449302 & 7 & 0,4553499 & 10,09295449 & 0,0012 \\
A: pH & 2,468505985 & 1 & 2,468505985 & 54,71510712 & $<0,0001$ \\
B: dosis de enzima & 0,013203125 & 1 & 0,013203125 & 0,29265086 & 0,6017 \\
C: tiempo de & 0,0242406 & 1 & 0,0242406 & 0,537299498 & 0,4822 \\
reacción & 0,373383654 & 1 & 0,373383654 & 8,276150324 & 0,0183 \\
A $^{2}$ & 0,03367225 & 1 & 0,03367225 & 0,746354587 & 0,4101 \\
AB & 0,041555314 & 1 & 0,041555314 & 0,921084853 & 0,3623 \\
AC & 0 & 1 & 0 & 0 & 1,0000 \\
BC & 0,406040581 & 9 & 0,04511562 & & \\
Residual & 0,406040581 & 4 & 0,101510145 & & \\
Falta de ajuste & 0 & 5 & 0 & & \\
Error puro & 3,593489882 & 16 & & & \\
Total corregido & & & & &
\end{tabular}

De acuerdo con la tabla 1 , el modelo tiene una significancia de 0,0012 y el término $\mathrm{A}$ o $\mathrm{pH}$ es la variable más significativa con un valor $P$ igual a 0,0001 , el cual cumple con las condiciones que permiten aceptar o rechazar la hipótesis [17], es decir, se rechaza la hipótesis nula donde las variables tiendan a dar el mismo resultado sin importar el valor de las variables. Por lo tanto, los factores o variables de proceso hacen que los resultados de los ensayos difieran dependiendo de estos, de modo que el $\mathrm{pH}$ es la variable que afecta con mayor intensidad el rendimiento de la hidrólisis alcalina enzimática de VCC.

\section{Efecto de las variables analizadas}

Los resultados experimentales denotan que tanto el $\mathrm{pH}$ como la dosis de enzima Blue $\mathrm{G}$ cumplen un papel importante para la hidrólisis de colágeno, tal y como se puede ver en la figura 1, mientras que el tiempo de reacción no permite determinar una influencia significativa comparada con el efecto de las variables $\mathrm{pH}$ y dosis de enzima.

La figura 1 representa la interacción de la variable de respuesta (concentración en $\mathrm{kg} / \mathrm{m}^{3}$ ) en el eje $\mathrm{Y}$, respecto a las variables de proceso tanto de $\mathrm{pH}$ como de dosis de enzima en el eje X. Los triángulos que se detallan en la gráfica hacen referencia a la mayor dosis; los cuadrados representan la menor dosis de enzima implementada en los ensayos y los círculos indican los rangos de $\mathrm{pH}$ que se emplearon. Se observa cómo las variables o factores $\mathrm{pH}$ y dosis de enzima interactúan en el proceso de obtención de colágeno hidrolizado, de modo que, para mayor dosis de enzima, la concentración de colágeno hidrolizado en la solución se incrementa con el pH, lo que hace más relevante el efecto para la mayor dosis de enzima empleada en los experimentos. Los resultados de los ensayos permiten evidenciar que la hidrólisis es más efectiva a $\mathrm{pH}$ alcalino, para valores de $\mathrm{pH}$ mayores a 8,5 en los ensayos realizados. Esto indica que el proceso es efectivo al realizarse en condiciones de hidrólisis alcalinaenzimática, donde da lugar a la degradación del material (VCC) en su mayor proporción teniendo en cuenta sus características para lograr la obtención del colágeno hidrolizado [9].

La figura 2 corresponde a la superficie de respuesta obtenida a partir de los resultados experimentales. El tiempo de reacción para el cual fue realizada la gráfica fue de 12,5 horas, debido a que se encontró que el efecto del tiempo de reacción dentro del rango de tiempos analizados no afecta de manera significativa la concentración final de colágeno hidrolizado en el producto de la reacción. 


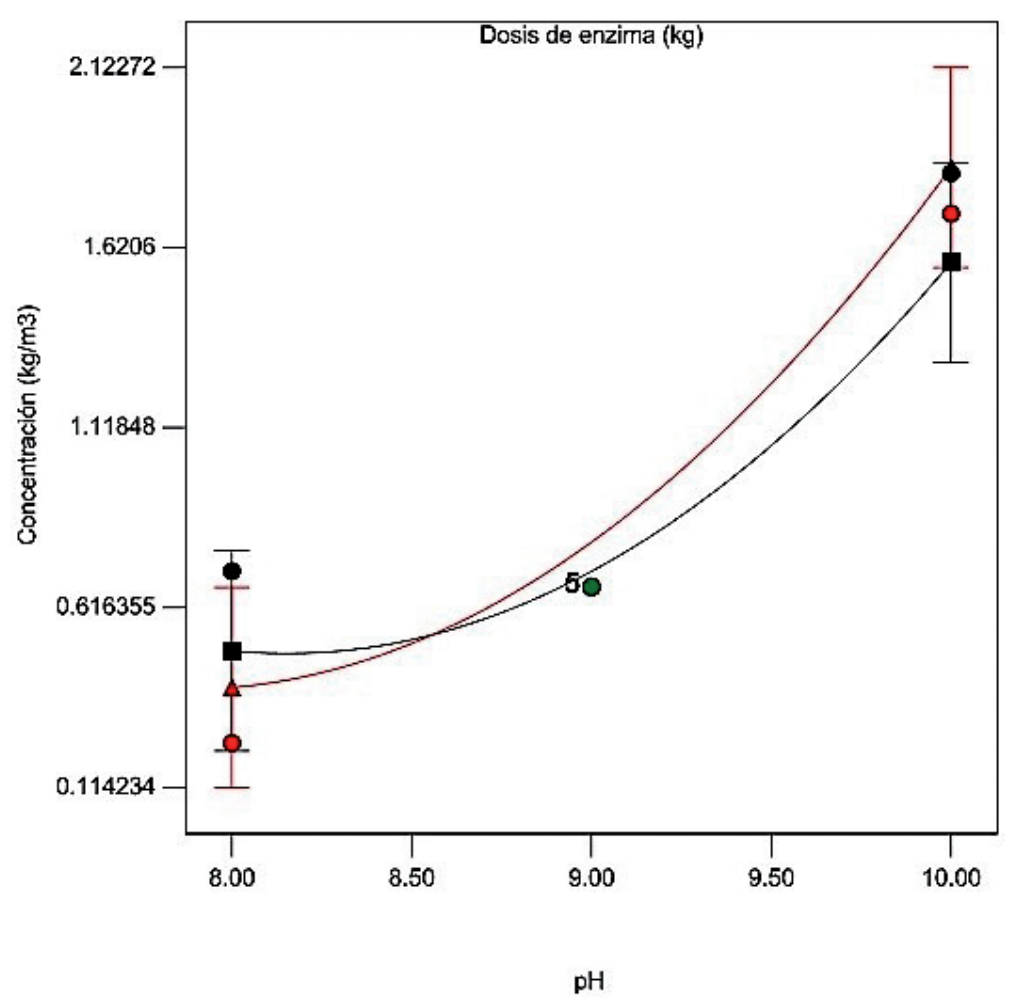

Figura 1. Interacción de las variables en la extracción de proteína hidrolizada.

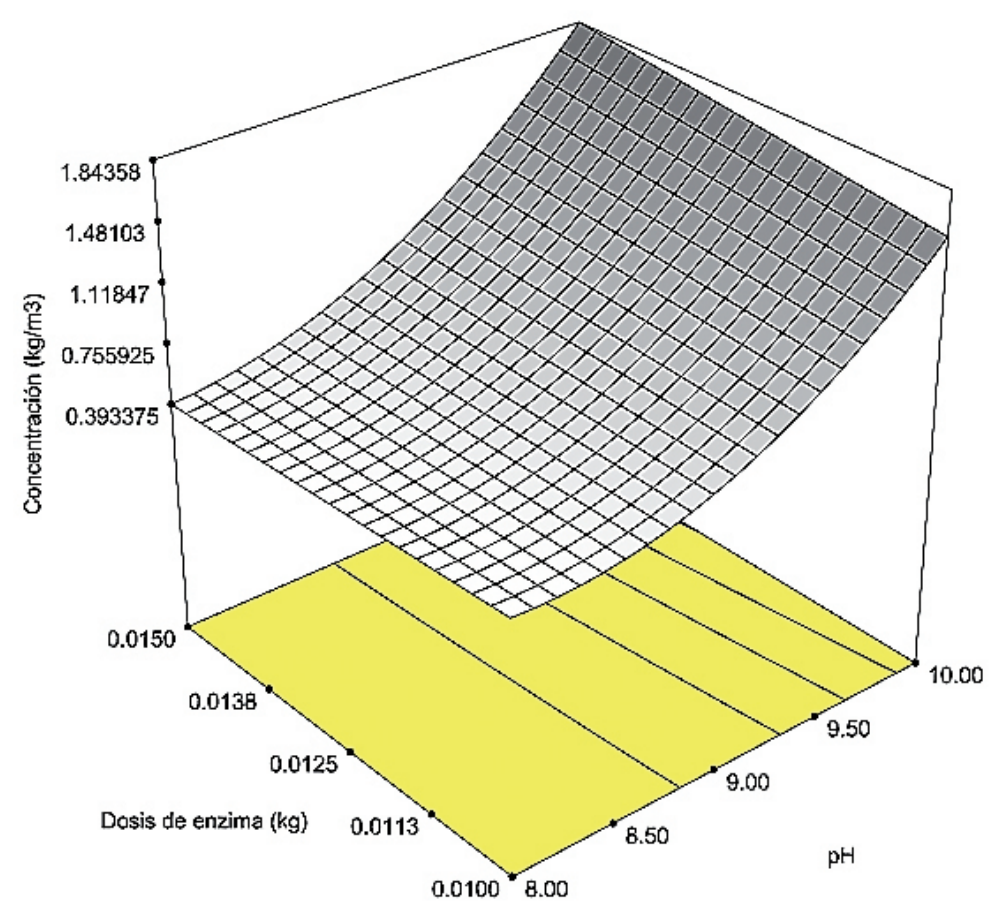

Figura 2. Condiciones óptimas de reacción. 
En la figura 2 se evidencia la variable de respuesta (concentración en $\mathrm{kg} / \mathrm{m}^{3}$ ) en el eje $Z$ respecto a las variables de proceso, tanto de $\mathrm{pH}$ como de dosis de enzima $(\mathrm{kg})$ en los ejes $\mathrm{Y}, \mathrm{X}$ respectivamente. Se observa que el aumento del $\mathrm{pH}$ para todas las dosis de enzima dio lugar al aumento en la concentración de colágeno hidrolizado obtenido, lo cual confirma lo descrito en la gráfica de interacción. El efecto del pH sobre el rendimiento de la reacción de hidrólisis enzimática es más significativo a $\mathrm{pH}$ alcalino y altas dosis de enzima. La ecuación obtenida para la superficie de respuesta es:

Concentración $=-0,75395-2,56370 * \mathrm{pH}-314,0500 *$ Dosis de enzima $+2,05020 *$ Tiempo de reacción $+0,31963 * \mathrm{pH}^{2}+$ $36.7000 * \mathrm{pH}^{*}$ Dosis de enzima-0,24120* $\mathrm{pH}^{*}$ Tiempo de reacción $+7,17204^{-12} *$ Dosis de enzima*Tiempo de reacción

El coeficiente de correlación $\left(R^{2}\right)$ para esta superficie de respuesta presentó un valor de 0,8870, es decir que existe un ajuste aceptable de los datos experimentales a los datos de la correlación de la superficie de respuesta. Con la ecuación de la superficie de respuesta se determinan los valores de las variables o factores que permitirían alcanzar la mayor concentración de colágeno hidrolizado dentro de los rangos de las variables seleccionadas. El resultado de esta optimización fue que a $\mathrm{pH}$ 10 , dosis de enzima $0,000015 \mathrm{~kg}$ y 12,5 horas de reacción, se puede alcanzar una concentración de colágeno hidrolizado de $1,828 \mathrm{~kg} / \mathrm{m}^{3}$, como condición óptima de proceso. El porcentaje másico de colágeno hidrolizado presente en la muestra de VCC con mayor concentración final del producto de interés es de $9,14 \%$.

\section{Análisis de electroforesis en gel SDS- poliacrilamida}

El análisis de electroforesis en gel SDSpoliacrilamida revela las bandas de corrido de muestras mediante la tinción de la proteína presente en el ensayo [21]. En la figura 3 se observa el gel de electroforesis correspondiente.

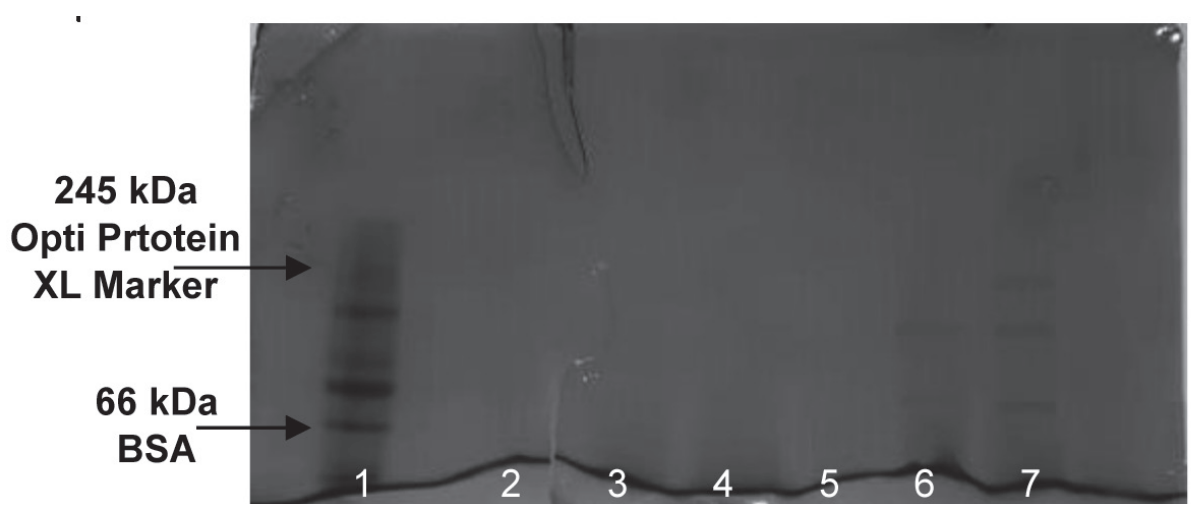

Figura 3. Gel de electroforesis poliacrilamida. SDS-PAGE.

En la figura 3 , el carril 1 revela el patrón o control de muestra seleccionado con el fin de determinar la presencia de proteínas en los ensayos; los carriles 2 a 7 hacen referencia a las muestras corridas de colágeno hidrolizado a partir de VCC. Dentro de ellos, los carriles 6 y 7 corresponden a las muestras que reportan mayor concentración de colágeno hidrolizado, ,es decir, para los cuales se da mayor tinción de la proteína y se observa la presencia de bandas que permiten la caracterización de colágeno [22], el cual tiene un peso molecular de $300 \mathrm{kDa}$
[23]. Por otra parte, las columnas en las cuales no se evidencia mayor tinción, se debe a que en el ensayo o muestra no presentan un cambio significativo en su estructura a partir del proceso de hidrólisis alcalina enzimática, lo cual evita la migración de las proteínas presentes; teniendo en cuenta estudios relacionados en los cuales se ha empleado la electroforesis en gel de poliacrilamida SDS-PAGE en la determinación de colágeno, se logra evidenciar que el colágeno tipo I se encuentra de forma más habitual [24]. 


\section{Conclusiones}

De acuerdo con los resultados del estudio realizado, se observó que la hidrólisis enzimática del colágeno presente en la VCC se lleva a cabo con mayor intensidad o, en otros términos, permite una mayor recuperación de colágeno hidrolizado, cuando el $\mathrm{pH}$ del medio es alcalino $(10)$ y permanece constante, la dosis de enzima es $0,000015 \mathrm{~kg}$ y se requieren 12,5 horas de tiempo de reacción; como resultado se obtiene una concentración de $1,828 \mathrm{~kg} / \mathrm{m}^{3}$, lo cual corresponde al 9,14 \% de colágeno hidrolizado por kg de VCC empleado. El análisis estadístico demostró que el $\mathrm{pH}$ representa la variable más relevante dentro del proceso de obtención de la proteína hidrolizada e interactuando al ser más alcalino y al aumentar la dosis de enzima; por otra parte, el tiempo de reacción no representa mayor importancia dentro de la hidrólisis. A partir de los resultados de los análisis, se puede confirmar que las VCC son susceptibles de hidrólisis para la extracción de colágeno hidrolizado, el cual puede ser utilizado en diferentes sectores comerciales. Se puede plantear que la hidrólisis alcalina-enzimática es una alternativa factible de ejecución por los integrantes del sector curtidor como tratamiento para el manejo de VCC, con lo cual se puede mitigar la contaminación ambiental al reducir la disposición final inadecuada causada por el sector e incentivar al tratamiento y valorización de residuos de este tipo.

\section{Referencias bibliográficas}

[1] Reyes Mena CM. Recuperación de Colágeno Libre de Cromo de los Residuos Sólidos Postcurtición en la Industria del Cuero. Quito; 2016.

[2] Secretaría Distrital de Ambiente, Guía de producción más limpia para el sector curtiembres de Bogotá Enfoque en vertimientos y residuos; 2015.

[3] Carrion N, Macias A. Formulación del plan de gestión integral de residuos peligrosos e implementación del registro para la curtiembre Galindo del PIESB; 2008.

[4] Rojas Amaya Z. F. V. Estudio económicofinanciero del aprovechamiento de las grasas extraídas del residuo de descarne 'unche' derivado del proceso de curtición en el municipio de Villapinzon-Cundinamarca. Bogotá D. C: 2010.
[5] Díaz A, Jiménez J, Pérez $M$, Narváez PC. Planteamiento y evaluación de las aplicaciones de los productos obtenidos en la hidrólisis alcalina productos obtenidos en la hidrólisis alcalina de las virutas de cromo generadas durante el procesamiento del cuero. Ing. e Investig. 2006;26(3):50-7.

[6] Lacoste A, Prez L. Obtención de un adhesivo como sustituto de materiales ureicos. vol. XI, pp. 1-11; 2007.

[7] Ferreira MJ, Almeida MF. Recycling of Leather Waste Containing Chromium -A Review. Sci. Technol. 2004;5(4):1-39.

[8] Cabeza LF, et al. Processing of leather waste: Pilot scale studies on chrome shavings. Isolation of potentially valuable protein products and chromium. Waste Manag. 1998;18(3):211-8.

[9] Jordán Núñez MF. Obtención de colágeno por hidrólisis alcalina-enzimática del residuo de "Wet Blue" en el proceso de curtición. Riobamba- Ecuador; 2011.

[10]Nieto A, Pedreza A. Evaluación del proceso enzimático para la producción de hidrolizado de colágeno a partir de las virutas de cuero "Wet Blue". Universidad de Bogotá Jorge Tadeo Lozano; 2017.

[11] INESCOP. Hidrólisis enzimática de rebajaduras de cuero WET-BLUE para la recuperación de agentes colagénicos; 2015.

[12] Benítez R, Albert I, Pagan J. Acta bioquímica clínica latinoamericana. Acta bioquímica clínica Latinoam. 2008;42(2):227-36.

[13] Goicoechea MC, De Zan MM, Candioti HLV. Metodología de la superficie de respuesta (rsm) diseño experimental y optimización de sistemas con múltiples respuestas; 2016.

[14] Genencor International Inc. Genencor Blue G Enzyme; 2004.

[15] Bradford MM. A Rapid and Sensitive Method for the Quantitation of Microgram Quantities of Protein Utilizing the Principle of Protein-Dye Binding; 1976.

[16] Vicéns J, Ainhoa O, Sánchez H, Medina Moral E. Análisis de Varianza (ANOVA); 2005.

[17] Gutierrez Pulido H. and De la Vara Salazar R. Análisis y Diseño de Experimentos, Segunda ed.; 2007.

[18] Lomonte Vigliotti B. Manual de Métodos Inmunológicos; 2007. 
[19]Applied Biological Materials Inc. Manual Instruction: Protein Markers-G266. Opti-Protein XL Marker; 2018. [Online]. Available: https:// www.abmgood.com/Prestained-Color-ProteinLadder-Broad-Range.html\#references. [Accessed: 14-Mar-2019].

[20] Escuela de Química. Hoja de seguridad Albúmina de suero bovino MSDS; 2016.

[21] García Pérez HM. Electroforesis en geles de poliacrilamida: fundamentos, actualidad e importancia. pp. 31-41; 2000.

[22] Rouzaud-Sández O, Pacheco-Aguilar R, Sotelo-Mundo RR, Ezquerra-Brauer JM, Torres-Arreola W. Caracterización parcial del colágeno extraído a partir del manto, aleta y tentáculos de calamar gigante (Dosidicus gigas) Partial characterization of collagen from mantle, fin, and arms of jumbo squid. (Dosidicus gigas). Cienc. y Tecnol. Aliment. 2009;6(2):101-8.

[23] Gómez Lizárraga K, Piña Barba C, Rodríguez Fuentes N. Obtención y caracterización de colágena tipo I a partir de tendón bovino. Superf. y Vacío. 2011;24(4):137-40.

[24] Serrano Gaona JC. Estandarización de un proceso de extracción de colágeno a partir de los residuos de fileteo de tilapia (Oreochromis sp) y cachama (Piaractus brachypomus) / Standardization of a process for extracting collagen from filleting waste from tilapia (Oreochromis) p. 85; 2011. 